\section{Electrotransport of Lattice Defects in Lithium Metal}

\section{P. Thernquist and A. Lodding}

Physics Department, Chalmers University of Technology, Gothenburg, Sweden

(Z. Naturforsch. 23 a, 627-628 [1968] ; received 20 February 1968)

The motion of lattice markers has been observed on $\mathrm{Li}$ specimens exposed to strong direct currents. The effective valence $z^{*}$ of a diffusing atom has been found to be about -1.8 at $90{ }^{\circ} \mathrm{C}$ and -1.2 at $160^{\circ} \mathrm{C}$. The defect resistivity (computed from Huntington's formula) is $0.62 \mu \Omega \mathrm{cm} / \%$ defects, and nearly independent of temperature. An Arrhenius plot yields an "activation energy" of electrotransport

$$
Q_{\mathrm{ET}}=12.9 \pm 0.4 \mathrm{kcal} / \mathrm{mole} \text {, }
$$

which is nearly equal to that of self-diffusion.

Preliminary results of electrotransport measurements in lithium have been reported at the conference "Electromigration and Thermal Diffusion in Metals", Münster 1965. Short notes have since appeared in this journal on bulk thermotransport ${ }^{1}$ and isotope thermotransport ${ }^{2}$ in lithium. A note on self-diffusion in $\mathrm{Li}^{3}$ will appear shortly. While the final discussion of the entire atom transport investigation in pure $\mathrm{Li}$ will be presented later this year, the experimental data on electrotransport ("self-transport") can be given here.

The experimental method is similar to those used by Wever ${ }^{4,5}$, Huntington ${ }^{6,7}$ and Lodding ${ }^{8}$. Lithium samples, in the form of rods, were force-cooled at both ends, while strong direct currents were passed through. The motion of inert surface markers at the hot portion of the samples was measured while similar markers at the cold ends served as a reference. Marker movement

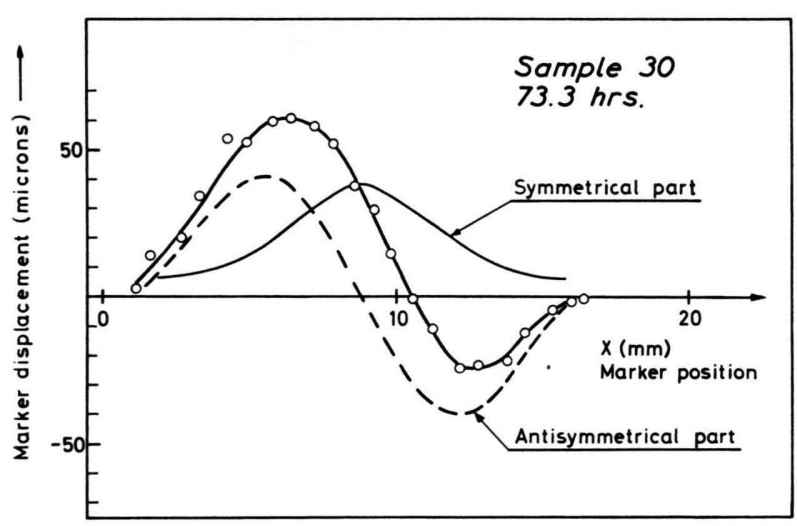

Fig. 1. Marker displacement for Li 30 sample after 73,3 hours.

$$
T_{\max }=136^{\circ} \mathrm{C} \text {. }
$$

1 A. Lodding and P. Thernquist, Z. Naturforsch. 21 a, 857 [1966].

2 P. Thernquist and A. Lodding, Z. Naturforsch. 22 a, 837 [1967].

3 A. Otт, J. N. Mundy, L. Löwenberg, and A. Lodding, to be published in this paper.

4 H. Wever, Z. Elektrochem. 60, 1170 [1956]. for the Li 30 sample is displayed in Fig. 1, where the correction is also shown for the uniquely great thermotransport effect occuring in $\mathrm{Li}$. The main modification from the earlier measurements of thermotransport ${ }^{1}$ was that the experiments were performed under a vacuum of $10^{-6}$ torr, instead of under an argon atmosphere.

Temperature measurements were obtained by means of five thermocouples embedded in the specimen. Two of them were electrically insulated to avoid contributions to the thermo-emf:s from the voltage drop across the $\mathrm{Li}$ rod. When passing alternating current along the samples before the direct current measurements, these contributions were not present and the temperature distribution could thus be obtained. In the calculations we used the velocities of markers near the insulated thermocouples, where the temperature was known with reasonable certainty.

It can be shown that the marker velocity, $v_{\mathrm{m}}$, is oppositely directed and proportional to the average ion drift velocity, $v_{\mathrm{i}}$, i. e.:

$$
v_{\mathrm{m}}=-\alpha v_{\mathrm{i}} .
$$

$\alpha$ is called the isotropy factor. Applying elasticity theory, Penney ${ }^{9}$ has shown that $\alpha$ depends on the geometry of the sample in such a way that for a thick, short sample $\alpha$ is close to unity (uniaxial transport) and for a thin, long one $\alpha$ is $1 / 3$ (isotropic transport). a can be obtained by comparing the total marker displacement with the change in dimensions of the sample $^{2}$. Our specimens proved to be an intermediate case, with $\alpha$-values between 0.47 and 0.62 , see Table 1 . $\alpha$ was measured at about $0.2 \mathrm{~mm}$ :s interval along each sample. The average value for each specimen was then used in the calculations.

\begin{tabular}{ccccc}
\hline Sample & $\begin{array}{c}T \\
\text { (averages of values } \\
\text { in Fig. 3) } \\
\end{array}$ & $\begin{array}{c}a \\
\text { (average) }\end{array}$ & $\begin{array}{c}-z^{*} \\
\text { (average) }\end{array}$ & $\begin{array}{c}\varrho^{*} \\
\text { (average) } \\
\mu \Omega \cdot \mathrm{cm}\end{array}$ \\
\hline 25 & 130 & 0.62 & 1.21 & 0.589 \\
$\%$ def.
\end{tabular}

Table 1 .

The results of electrotransport experiments are usually given in terms of the "effective valence", $z^{*}$, of a diffusing atom. $z^{*}$ is defined by the Nernst-Einsteintype relation

$$
v_{\mathrm{i}}=\left(D^{*} E e z^{*}\right) / f k T
$$

5 H. Hering and H. Wever, Acta Met. 15, 377 [1967].

6 H. B. Huntington and A. R. Grone, J. Phys. Chem. Solids 20, 76 [1961].

7 P. S. C. Ho and H. B. Huntington, ibid. 27, 1319 [1966].

8 A. Lodding, ibid. 26, 143 [1965].

9 R. V. Penney, J. Phys. Chem. Solids 25, 335 [1964]. 
which can be written

$$
v_{\mathrm{m}}=-\alpha\left(D^{*} j \varrho e z^{*}\right) / f k T .
$$

Here $D^{*}$ is the self-diffusion coefficient as determined by tracer experiments, $f$ the Bardeen-Herring correlation factor $(0.7272$ for vacancy mechanism in bcc metals), $k$ Boltzmann's constant, $T$ absolute temperature, $e$ the electronic charge, $E$ the electric field, $j$ the current density (order of magnitude $2 \cdot 10^{3} \mathrm{~A} / \mathrm{cm}^{2}$ in our experiments) and $\varrho$ the resistivity of the metal. As can be seen from formula (2), the temperature dependence of $z^{*}$ being much smaller than that of $D^{*}$, a semilogarithmic plot of $\left(v_{\mathrm{m}} T\right) / j$ vs. $1 / T$ should give a nearly straight line, the slope of which should, if the transport mechanisms are identical, yield an "activation energy" similar to that of self-diffusion. Such a plot for our data is shown in Fig. 2 and a least square fit of the data yields $Q_{\mathrm{ET}}=12.9 \pm 0.4 \mathrm{kcal} / \mathrm{mole}$ which is, within the error limits, equal to the preliminary value obtain- ${ }^{*}$ ed by Отт et al. ${ }^{10}, Q_{\mathrm{D}}=12.6 \pm 0.3 \mathrm{kcal} / \mathrm{mole}$.

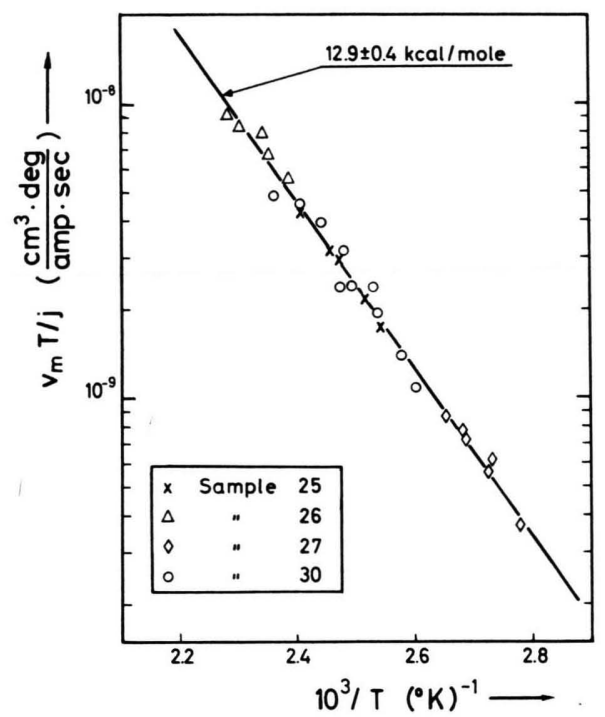

Fig. 2. Arrhenius plot for electrotransport in Li. Data from four samples. The straight line yields an "activation energy" of $12.9 \pm 0.4 \mathrm{kcal} / \mathrm{mole}$.

According to a theory, given by Huntington ${ }^{6,11}$ (see also Ref. $\left.{ }^{12}\right) z^{*}$ can be expressed by the formula

$$
z^{*}=z\left\{q^{*} / q^{-\frac{1}{2}}\left(m^{*} /\left|m^{*}\right|\right)\left(\varrho^{*} / \varrho\right)\right\},
$$

where $z$ is the number of free electrons per metal ion, $q^{*}$ and $q$ the degree of ionization of a diffusing atom and an atom at a lattice site, respectively, $m^{*}$ the effective mass of the electron and $\varrho^{*}$ the "effective defect

10 A. OTт, private communication.

11 H. B. Huntington and P. S. C. Ho, J. Phys. Soc. Japan 18 , Suppl. 2, 202 [1963]. resistivity". In Fig. $3 z^{*}$ and $\varrho^{*}$ are plotted against $1 / \varrho$ and $T$, where we have assumed $z=1$ and $m^{*}=\left|m^{*}\right|$ (justifiable in the alkalis), and $q^{*} / q=1$ (reasonable for self-transport).

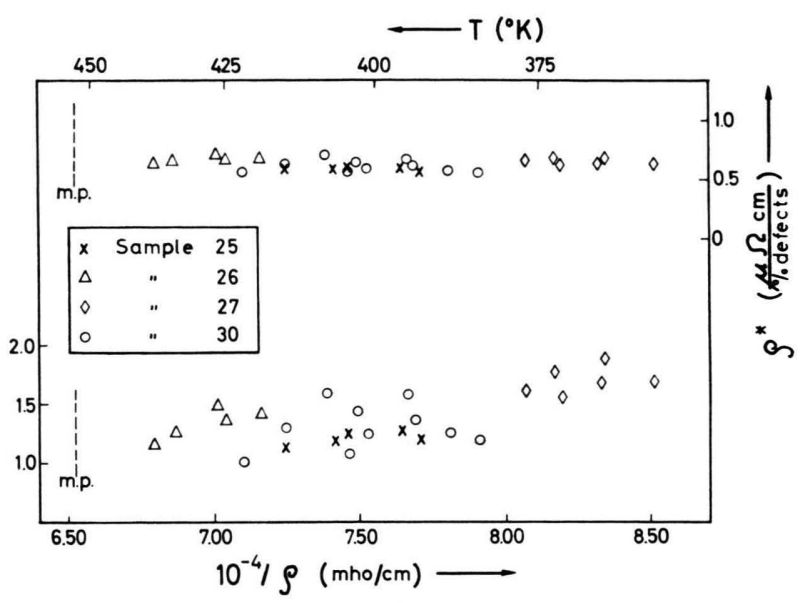

Fig. 3. Effective valence and effective defect resistivity as functions of (resistivity) ${ }^{-1}$ and of temperature.

From Fig. 3 we conclude that $\left(-z^{*}\right)$ is slowly decreasing with temperature. It must be emphasized, however, that errors in the $a$ determination play an important role for such considerations, as seen in Fig. 3. Thus the measured $\alpha$ of sample 25 seems to be too great (see also Table 1). Also, the temperature dependence of $z^{*}$ is sensitive to the activation energy of selfdiffusion, which has at present not yet been determined with final accuracy.

The defect resistivity, $\varrho^{*}$, has the average value of $0.62 \mu \Omega \mathrm{cm} / \%$ defects. This can be compared with SulLIVAN's ${ }^{13}$ result in sodium: $0.44 \mu \Omega \mathrm{cm} / \%$ defects. Both of these are considerably lower than expected from theory. The reason for this is still not quite obvious.

Fig. 3 also shows that $\varrho^{*}$ is essentially constant over the temperature range studied. In most earlier non-isothermal marker-type experiments it was found that $\varrho^{*}$ decreased with increasing temperature. In some such earlier investigations a certain lack of reproducibility could also be detected. Measurements in $\mathrm{In}^{8}$ and $\mathrm{Ag}^{7}$ gave, however, essentially constant $\varrho^{*}$, as did the most recent isothermal investigation of $\mathrm{Au}^{14}$.

This research has been supported by Statens Naturvetenskapliga Forskningsråd and Swedish Council for Applied Research. - Fil. mag. A.-M. Joserson and ing. H. Olsson assisted us skilfully during several stages of the work.

12 A. Lodding, Z. Naturforsch. 14 a, 939 [1959].

13 G. A. Sullivan, Phys. Rev. 154, 605 [1967].

14 H. M. Gilder and D. Lazarus, Phys. Rev. 145, 507 [1966]. 